\title{
On hyperballeans of bounded geometry
}

\author{
Igor Protasov and Ksenia Protasova
}

April 25, 2018

\begin{abstract}
A ballean (or coarse structure) is a set endowed with some family of subsets, the balls, is such a way that balleans with corresponding morphisms can be considered as asymptotic counterparts of uniform topological spaces. For a ballean $\mathcal{B}$ on a set $X$, the hyperballean $\mathcal{B}^{b}$ is a ballean naturally defined on the set $X^{b}$ of all bounded subsets of $X$. We describe all balleans with hyperballeans of bounded geometry and analyze the structure of these hyperballeans.
\end{abstract}

MSC 54E35, 51F99

Key words. ballean, hyperballean, coarse equivalence, bounded geometry, Cantor macrocube.

\section{Introduction and preliminaries}

Following [7], [8], we say that a ball structure is a triple $\mathcal{B}=(X, P, B)$, where $X, P$ are non-empty sets, and for all $x \in X$ and $\alpha \in P, B(x, \alpha)$ is a subset of $X$ which is called a ball of radius $\alpha$ around $x$. It is supposed that $x \in B(x, \alpha)$ for all $x \in X, \alpha \in P$. The set $X$ is called the support of $\mathcal{B} P$ is called the set of radii.

Given any $x \in X, A \subseteq X, \alpha \in P$, we set

$$
B^{*}(x, \alpha)=\{y \in X: x \in B(y, \alpha)\}, \quad B(A, \alpha)=\bigcup_{a \in A} B(a, \alpha) .
$$

A ball structure $\mathcal{B}=(X, P, B)$ is called a ballean if

- for any $\alpha, \beta \in P$, there exist $\alpha^{\prime}, \beta^{\prime} \in P$ such that, for every $x \in X$,

$$
B(x, \alpha) \subseteq B^{*}\left(x, \alpha^{\prime}\right), \quad B^{*}(x, \beta) \subseteq B\left(x, \beta^{\prime}\right) ;
$$

- for every $\alpha, \beta \in P$, there exist $\gamma \in P$ such that, for every $x \in X$,

$$
B(B(x, \alpha), \beta) \subseteq B(x, \gamma)
$$


- for any $x, y \in X$, there exists $\alpha \in P$ such that $y \in B(x, \alpha)$.

We note that a ballean can be considered as an asymptotic counterpart of a uniform space, and could be defined [9] in terms of entourages of the diagonal $\Delta_{X}$ in $X \times X$. In this case a ballean is called a coarse structure. For categorical look at the ballean and coarse structures as "two faces of the same coin" see [4].

Let $\mathcal{B}=(X, P, B), \mathcal{B}^{\prime}=\left(X^{\prime}, P^{\prime}, B^{\prime}\right)$ be balleans. A mapping $f$ : $X \longrightarrow X^{\prime}$ is called coarse if, for every $\alpha \in P$, there exists $\alpha \prime \in P^{\prime}$ such that

$$
f(B(x, \alpha)) \subseteq B^{\prime}\left(f(x), \alpha^{\prime}\right) .
$$

A bijection $f: X \longrightarrow X^{\prime}$ is called an asymorphism between $\mathcal{B}$ and $\mathcal{B}^{\prime}$ if $f$ and $f^{-1}$ are coarse mappings. In this case $\mathcal{B}$ and $\mathcal{B}^{\prime}$ are called asymorphic. If $X=X^{\prime}$ and the identity mapping $i d: X \longrightarrow X^{\prime}$ is an asymorphism, we identify $\mathcal{B}$ and $\mathcal{B}^{\prime}$, and write $\mathcal{B}=\mathcal{B}^{\prime}$. Given any ballean $\mathcal{B}=(X, P, B)$, replacing each ball $B(x, \alpha)$ to $B(x, \alpha) \cap B^{*}(x, \alpha)$, we get the same ballean, so in what follows we suppose that $B(x, \alpha)=B^{*}(x, \alpha)$.

Let $\mathcal{B}=(X, P, B)$ be a ballean. Each non-empty subset $Y$ of $X$ defines a subballean $\mathcal{B}_{Y}=\left(X, P, B_{Y}\right)$, where $B_{Y}(y, \alpha)=Y \cap B(y, \alpha)$. A subset $Y$ is called large if $X=B(Y, \alpha)$ for some $\alpha \in P$. Two balleans $\mathcal{B}$ and $\mathcal{B}^{\prime}$ with the support $X$ and $X^{\prime}$ are called coarsely equivalent if there exist large subsets $Y \subseteq X$ and $Y^{\prime} \subseteq X^{\prime}$ such that the balleans $\mathcal{B}_{Y}$ and $\mathcal{B}_{Y^{\prime}}^{\prime}$ are asymorphic.

For a ballean $\mathcal{B}=(X, P, B)$, a subset $Y$ of $X$ is called bounded if there exist $x \in X$ and $\alpha \in P$ such that $Y \subseteq B(x, \alpha)$. A ballean $\mathcal{B}$ is called bounded if the support $X$ is bounded. Each bounded ballean is coarsely equivalent to a ballean whose support is a singletone.

Now we are ready to introduce the main subject of the note. For a ballean $\mathcal{B}=(X, P, B)$, we denote by $X^{b}$ the family of all non-empty bounded subsets of, consider the ballean $\mathcal{B}^{b}=\left(X^{b}, P, B^{b}\right)$, where

$$
B^{b}(Y, \alpha)=\left\{Z \in X^{b}: Z \subseteq B(Y, \alpha), Y \subseteq B(Z, \alpha)\right\},
$$

and say that $\mathcal{B}^{b}$ is the hyperballean of $\mathcal{B}$.

For $\alpha \in P$, a subset $S$ of $X$ is called $\alpha$-discrete if $B(x, \alpha) \cap S=\{x\}$ for each $x \in S$. We say that $\mathcal{B}$ is of bounded geometry if there exist $\alpha \in P$ and a function $f: P \longrightarrow \mathbb{N}$ such that if $S$ is an $\alpha$-discrete subset of a ball $B(x, \beta)$ then $|\delta| \leq f(\beta)$. A ballean $\mathcal{B}$ is called uniformly locally finite if, for every $\beta \in P$, there is $n(\beta) \in \mathbb{N}$ such that $|B(x, \beta)| \leq n(\beta)$ for every $x \in X$. By [6], $\mathcal{B}$ is of bounded geometry if and only if there exists large subset $Y$ of $X$ such that $\mathcal{B}_{Y}$ is uniformly locally finite.

It should be mentioned that the notion of bounded geometry went from asymptotic topology where metric spaces of bounded geometry 
play the central part [5]. For interrelations between balleans of bounded geometry and $G$-spaces see [6].

Every metric space $(X, d)$ defines the metric ballean $\left(X, \mathbb{R}^{+}, B_{\alpha}\right)$, where $B_{d}(x, r)=\{y \in X: d(x, y) \leq r\}$. A ballean $\mathcal{B}$ is called metrizable if $\mathcal{B}$ is asymorphic to some metric ballean. By [ 8 , Theorem 2.1.1], for a ballean $\mathcal{B}$, the following statements are equivalent: $\mathcal{B}$ is metrizable, $\mathcal{B}$ is coarsely equivalent to some metrizable ballean, the set $P$ has a countable confinal subset $S$. We recall $S$ is confinal if, for every $\beta \in P$ there is $\alpha \in S$ such that $\alpha>\beta$. Here $\alpha>\beta$ means that $B(x, \beta) \subseteq B(x, \alpha)$ for each $x \in X$. Applying this criterion, we conclude that, for every metrizable ballean $\mathcal{B}$, the hyperballean $\mathcal{B}^{b}$ is metrizable.

\section{Results}

For a non-empty set $X$ and the family $\mathcal{F}_{X}$ of all finite subsets of $X$, we denote by $\mathbf{F}_{X}$ the ballean $\left(X, \mathcal{F}_{X}, B_{\mathbf{F}}\right)$ where

$$
B_{\mathbf{F}}(x, F)= \begin{cases}\{x\} & \text { if } x \notin F ; \\ F & \text { if } x \in F .\end{cases}
$$

Then $\mathbf{F}_{X}^{b}=\left\{\mathfrak{F}_{X} \backslash\{\emptyset\}, \mathfrak{F}_{X}, B_{\mathbf{F}}^{b}\right\}$, where $B_{\mathbf{F}}^{b}(H, F)=\{H\}$ if $H \cap F=\emptyset$ and $B_{\mathbf{F}}(H, F)=\{(H \backslash F) \cup Z: Z \subseteq H, Z \neq \emptyset\}$ otherwise.

The ballean $\mathbf{F}_{\omega}, \omega=\{0,1 \ldots\}$ is metrizable (say, by the metric $d(m, n)=\left|2^{m}-2^{n}\right|$ ), so $\mathbf{F}_{\omega}^{b}$ is also metrizable (say, by the Hausdorff metric $\left.b_{H}\right)$. At the end of the note, we point out some more explicit metrization of $\mathbf{F}_{\omega}^{b}$.

Theorem 2.1. For an unbounded ballean $\mathcal{B}=(X, P, B)$, the following statements hold:

(i) $\mathcal{B}^{b}$ is uniformly locally finite if and only if $\mathcal{B}=\mathbf{F}_{X}$;

(ii) $\mathcal{B}^{b}$ is of bounded geometry if and only if there exists a large subset $Y$ of $X$ such that $\mathcal{B}_{Y}=\mathbf{F}_{Y}$.

For a cardinal $\kappa$, we denote by $\mathbf{Q}_{\kappa}$ the ballean with the support

$$
\mathbf{Q}_{\kappa}=\left\{\left(x_{\alpha}\right)_{\alpha<\kappa}: x_{\alpha} \in\{0,1\}, x_{\alpha}=0\right.
$$

for all but finitely many $\alpha<\kappa\}$,

the set of radii $\mathcal{F}_{\kappa}$ and the balls

$$
B_{\mathbf{Q}}\left(\left(x_{\alpha}\right)_{\alpha<\kappa}, F\right)=\left\{\left(y_{\alpha}\right)_{\alpha<\kappa}: x_{\alpha}=y_{\alpha} \quad \text { for all } \alpha \in \kappa \backslash F\right\} .
$$


The ballean $\mathbf{Q}_{\omega}$ is known as the Cantor macrocube and sometimes is denoted by $2^{<\omega}$ or $2^{<\mathbb{N}}$. For characterization of balleans coarsely equivalent to the Cantor macrocube see [3]. In [1], $2^{<\kappa}$ denotes the ballean of all $\{0,1\} \kappa$-sequences $\left(x_{\alpha}\right)_{\alpha<\kappa}$ such that $\left|\left\{\alpha<\kappa: x_{\alpha}=1\right\}\right|<\kappa$.

A ballean $\mathcal{B}=(X, P, B)$ is called asymptotically scattered if, for every unbounded subset $Y$ of $X$, there is $\alpha \in P$, such that, for every $\beta \in P$, there exists $y \in Y$ such that

$$
(B(y, \beta) \backslash B(y, \alpha)) \bigcap Y=\emptyset .
$$

For asymptotically scattered subbaleans of group balleans see [2].

For a ballean $\mathcal{B}=(X, P, B)$, the subset $Y, Z$ of $X$ are called close if there exists $\alpha \in P$ such that $Y \subseteq B(Z, \alpha), Z \subseteq B(Y, \alpha)$.

Theorem 2.2. Let $\kappa$ be an infinite cardinal, $n \in \mathbb{N},[\kappa]^{n}=\{F \subset \kappa$ : $|F|=n\}, x \in \kappa$. Then the following statements hold:

(i) the subbalean of $\mathbf{F}_{\kappa}^{b}$ with the support $[\kappa]^{n}$ is asymptotically scattered;

(ii) the subbalean of $\mathbf{F}_{\kappa}^{b}$ with the support $\left\{F \in \mathfrak{F}_{\kappa}: x \in F\right\}$ is asymorphic to $\mathbf{Q}_{\kappa}$;

(iii) $\mathbf{F}_{\omega}^{b}$ can be partitioned into countably many pairwise close Cantor macrocubes but $\mathbf{F}_{\omega}^{b}$ is not coarsely equivalent to $\mathbf{Q}_{\omega}$.

At the end of the note, we describe some explicit asymorphic embedding of $\mathbf{F}_{\omega}^{b}$ into $\mathbf{Q}_{\omega}$.

\section{Proofs}

Proof of Theorem 1.2. (i) By the definition of balls in $\mathbf{F}_{\omega}^{b}, \mathbf{F}_{\omega}^{b}$ is uniformly locally finite.

If the identity mapping $i d: X \longrightarrow X$ is not an asymorphism between $\mathcal{B}$ and $\mathbf{F}_{X}$ then we can choose $\alpha \in P$ and a sequence $\left(x_{n}\right)_{n<\omega}$ in $X$ such that $\left|B\left(x_{n}, \alpha\right)\right|>1$ and $B\left(x_{i}, \alpha\right) \bigcap B\left(x_{j}, \alpha\right)=\emptyset$ for all $i<j<\omega$. For each $i<\omega$, we pick $y_{i} \in B\left(x_{i}, \alpha\right), y_{i} \neq x_{i}$, put $X_{n}=\left\{x_{0}, \ldots, x_{n}\right\}$, $X_{n, i}=X_{n} \bigcup\left\{y_{i}\right\}, i \leq n<\omega$. Then $X_{n, i} \in B^{b}\left(X_{n}, \alpha\right)$, so $\left|B^{b}\left(X_{n}, \alpha\right)\right|>$ $n$ and $\mathcal{B}$ is not uniformly locally finite.

(ii) We assume that $Y$ is a large subset of $X$ and choose $\beta \in P$ such that $B(Y, \beta)=X$. For each $x \in X$, we pick $y_{x} \in Y$ such that $y_{x} \in B(x, \beta)$. If $F \in X^{b}$ then $\left\{y_{x}: x \in F\right\} \in Y^{b}$ and $F \in B^{b}\left(\left\{y_{\kappa}: x \in\right.\right.$ $F\}, \beta)$. It follows that $B^{b}\left(Y^{b}, \beta\right)=X^{b}, Y^{b}$ is large in $X^{b}$ so $\mathcal{B}_{Y}^{b}$ and $\mathcal{B}^{b}$ are coarsely equivalent. In particular, if $\mathcal{B}_{Y}=\mathbf{F}_{Y}$, we conclude that $\mathcal{B}$ is of bounded geometry. 
We suppose that $\mathcal{B}^{b}$ is of bounded geometry and let $\alpha \in P$ and $f: P \longrightarrow \mathbb{N}$ witness this property. Using Zorn's lemma, we choose a maximal by inclusion subset $Y$ of $X$ such that $B(y, \alpha) \bigcap B\left(y^{\prime}, \alpha\right)=\emptyset$ for all distinct $y, y^{\prime} \in Y$. We show that $\mathcal{B}_{Y}=\mathbf{F}_{Y}$.

If the identity mapping $i d: Y \longrightarrow Y$ is not an asymorphism between $\mathcal{B}_{Y}$ and $\mathbf{F}_{Y}$ then there are $\beta \in P$ and a sequence $\left(y_{n}\right)_{n \in \omega}$ in $Y$ such that $\left|B_{Y}\left(y_{n}, \beta\right)\right|>1$ and $B_{Y}\left(y_{i}, \beta\right) \bigcap B_{Y}\left(y_{j}, \beta\right)=\emptyset$ for all $i<j<\omega$. For each $i<\omega$, we pick $z_{i} \in B_{Y}\left(y_{i}, \beta\right), z_{i}=y_{i}$, put $Y_{n}=\left\{y_{0}, \ldots, y_{n}\right\}$, $Y_{n, i}=Y_{n} \bigcup\left\{y_{i}\right\}, i \leq n<\omega$. Then $Y_{n, i} \in B^{b}\left(Y_{n}, \beta\right)$ and the set $\left\{Y_{n, i}\right.$ : $i \leq n\}$ is $\alpha$-discrete. Thus, for $n>f(\beta)$ we get a contradiction with the choice of $\alpha$ and $f$.

Proof of Theorem 2.2. ( $i$ ) We say that a subset of a ballean is asymptotically scattered if corresponding subballean has this property. We use the following observation: the union of two asymptotically scattered subsets is asymptotically scattered (see [2]).

We note that every unbounded subset in $\mathbf{F}_{\kappa}^{b}$ is infinite and proceed on induction by $n$. For $n=1$, the statement is evident: given any $H \in \mathfrak{F}_{\kappa}$ and an infinite subset $Y$ of $[\kappa]^{1}$, we take $\{y\} \in Y, y \in H$ and get $B_{\mathbf{F}}(\{y\}, H)=\{y\}$.

Assuming that the statement is true for $[\kappa]^{n}$, let $Y$ be an infinite subset of $[\kappa]^{n+1}$. For each $F \in[\kappa]^{n+1}$, we denote by $\min F$ and $\max F$, the minimal and maximal elements of $F$ with respect to the ordinal ordering of $\kappa$ and consider two cases.

Case: the set $\{\min F: F \in Y\}$ is infinite. We take an arbitrary $H \in$ $\mathfrak{F}_{\kappa}$ and choose $F \in Y$ such that $\max H<\min F$. Then $B_{\mathbf{F}}^{b}(F, H)=\{F\}$.

Case: the set $\{\min F: F \in Y\}$ is finite, $\{\min F: F \in Y\}=$ $x_{1}, \ldots, x_{n}$. For each $i \in\{1, \ldots, n\}$, we denote $Z_{i}=\left\{F \in[\kappa]^{n+1}\right.$ : $\left.x_{i} \in F\right\}$. We note that $Z_{i}$ is asymorphic to $[\kappa]^{n}$ and, by the inductive assumption, $Z_{i}$ is asymptotically scattered.

Then $Z_{1} \cup \ldots \cup Z_{n}$ is asymptotically scattered, $Y \subseteq Z_{1} \cup \ldots \cup Z_{n}$ and we can use definition of asymptotically scattered subsets to choose $\alpha \in \mathfrak{F}_{X}$ suitable for $Y$.

(ii) We use the standard bijection $\chi: \mathfrak{F}_{\kappa} \longrightarrow Q_{\kappa}$ defined by $\chi(K)=$ $\left(x_{\alpha}\right)_{\alpha<\kappa}$, where $x_{\alpha}=1$ if and only if $\alpha \in K$. Then the restriction of $\chi$ to $\left\{F \in \mathfrak{F}_{\kappa}\right\}: x \in F$ is a asymorphic embedding. Indeed, to verity this property we may use as radii in $\mathbf{F}^{b}$ only balls containing $x$. Clearly, $\chi\left\{F \in \mathfrak{F}_{\kappa}: \kappa \in F\right\}$ is asymorphic to $\mathbf{Q}_{\kappa}$.

(iii) For every $n \in \omega$, let $\mathcal{M}_{n}=\left\{F \in \mathfrak{F}_{X}: \min F=n\right\}$. Applying (ii), we see that $\mathcal{M}_{n}$ is asymorphic to $\mathbf{Q}_{\omega}$. We take arbitrary $i, j \in \omega$, denote $m=\max \{i, j\}, I_{m}=\{0, \ldots, m\}$. Then $\mathcal{M}_{i} \subseteq B_{\mathbf{F}}^{b}\left(\mathcal{M}_{j}, I_{m}\right)$, $\mathcal{M}_{j} \subseteq B_{\mathbf{F}}^{b}\left(\mathcal{M}_{i}, I_{m}\right)$ so $\mathcal{M}_{i}, \mathcal{M}_{j}$ are close.

Given $H \in \mathfrak{F}_{\omega}$, we take $F \in \mathfrak{F}_{\omega}$ such that $\max H<\min F$. Then 
$B_{\mathbf{F}}^{b}(F, H)=\{F\}$. In terminology of [3], it means that $\mathbf{F}_{\omega}^{b}$ has an asymptotically isolated balls but every ballean coarsely equivalent to $\mathbf{Q}_{\omega}$ has no isolated balls.

To embed asymorphically $\mathbf{F}_{\omega}^{b}$ into $\mathbf{Q}_{\omega}$, we use $2 \mathbb{N}$ in place of $\omega$. We define a mapping $f: \mathfrak{F}_{2 \mathbb{N}}\{\emptyset\} \longrightarrow Q_{\omega}$ by the $f(K)=\left(x_{n}\right)_{n<\omega}$, where $x_{n}=1$ if and only if $n \in\{\min K-1\} \bigcup K$. We note that the set $S=f\left(\mathfrak{F}_{2 \mathbb{N}} \backslash\{\emptyset\}\right)$ consists of all sequences $\left(x_{n}\right)_{n<\omega}$ with at least two non-zero coordinates and such that the first non-zero coordinate of $\left(x_{n}\right)_{n<\omega}$ is odd and all other are even. For each $K \in \mathfrak{F}_{2 \mathbb{N}} \backslash\{\emptyset\}$ and $n \in \mathbb{N}$, we have

$$
f\left(B_{\mathbf{F}}(K,\{2,4, \ldots, 2 n\})=S \bigcap B_{\mathbf{Q}}(f(K),\{1,2 \ldots, 2 n\}),\right.
$$

witnessing that $f$ is an asymorphic embedding of $\mathbf{F}_{2 \mathbb{N}}^{b}$ into $\mathbf{Q}_{\omega}$.

With this representation, $\mathbf{F}_{\omega}^{b}$ can be easily metrizable by means of restriction to $S$ of the stadard metric $d$ on $Q_{\omega}: d\left(\left(x_{n}\right)_{n \in \omega},\left(y_{n}\right)_{n \in \omega}\right)=$ $\min \left\{m: x_{n}=y_{n} \quad\right.$ for all $\left.n \geq m\right\}$.

1. T. Banakh, I. Protasov, D. Repov $\breve{s}$, S. Slobodianiuk, Classifying homogeneous celular ordinal balleans up to coarse equivalence, preprint (arxiv: 1409.3910v2).

2. T. Banakh, I. Protasov, S. Slobodianiuk, Scattered subsets of groups, Ukr. Math. Zh. 67 (2015), P. 304-312.

3. T. Banakh, I. Zarichnyi, Characterizing the Cantor bi-cube in asymptotic categories, Groups, Geometry and Dynamics 5 (2011), P. 691-728.

4. D. Dikranjan, N. Zava, Some categorical aspects of coarse spaces and balleans, Topology Appl., to appear.

5. A. Dranishnikov, Asymptotic Topology, Russian Math. Surveys, 55 (2000), 1085-1129.

6. I. Protasov, Balleans of bounded geometry and G-spaces, Math. Stud. 30 (2008), 61-66.

7. I. Protasov, T. Banakh, Ball Structures and Colorings of Graphs and Groups, Math. Stud. Monogr. Ser. 11, Vol. 11, VNTL Publisher, Lviv, 2003.

8. I. Protasov, I. Zarichnyi, General Asymptology, Math. Stud. Monogr. Ser 12, Vol. 11, VNTL Publisher, Lviv, 2007. 
9. J. Roe,Lectures on coarse geometry, Univ. Lecture Series 31, American Mathematical Society, Providence, 2003.

\section{CONTACT INFORMATION}

Igor Protasov

Department of Cybernetics

Kyiv University

Prospect Glushkova 2, corp. 6

03680 Kyiv, Ukraine

e-mail: i.v.protasov@gmail.com

Ksenia Protasova

Department of Cybernetics

Kyiv University

Prospect Glushkova 2, corp. 6

03680 Kyiv, Ukraine

e-mail: k.d.ushakova@gmail.com 